Водные биоресурсы и среда обитания

2019, том 2, номер 3, с. 41-58

http://journal.azniirkh.ru, www.azniirkh.ru ISSN 2618-8147 print, ISSN 2619-1024 online

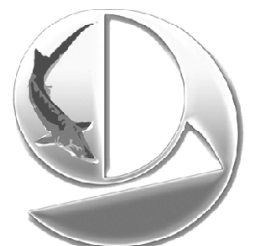

Aquatic Bioresources \& Environment 2019, vol. 2, no. 3, pp. 41-58

http://journal.azniirkh.ru, www.azniirkh.ru ISSN 2618-8147 print, ISSN 2619-1024 online

УДК 582.263+582.271(282.25)(549.1)

\title{
DIVERSITY AND SEASONAL DYNAMICS OF CHLOROPHYTA REICHENBACH, 1828 AND CHAROPHYTA MIGULA, 1890 ALGAE IN THE SWAT RIVER BASIN, PAKISTAN
}

\author{
(C) 2019 Asghar Ali ${ }^{1,2}$, Lal Badshah ${ }^{2}$, Sophia Barinova ${ }^{3 *}$ \\ ${ }^{1}$ Govt AKL Post Graduate College Matta, Swat, Pakistan \\ ${ }^{2}$ Department of Botany, University of Peshawar, Khyber Pakhtunkhwa, Old Jamrud Road, Pakistan, PK-25120 \\ ${ }^{3}$ Institute of Evolution, University of Haifa, Haifa 3498838, Israel \\ *E-mail:sophia@evo.haifa.ac.il
}

\begin{abstract}
Increasing water pollution and climatic changes affect algal populations and, in turn, regional ecosystems. The algal flora was assessed at five different localities of District Swat, Khyber Pakhtunkhwa, Pakistan at altitude 972-2,061 m a.s.l. Altogether, 105 taxa of algae from Charophyta and Chlorophyta divisions were found for the first time in 200 samples of algae collected during 2007-2016. The number of Chlorophyta species, represented by 86 taxa, strongly prevailed over Charophyta species (19 taxa). Water turbidity, water transparency, and iron have a positive correlation with altitude, whereas water temperature, $\mathrm{pH}$, total dissolved solids (TDS), ammonia, nitrate concentration, and zinc correlated negatively. Over $50 \%$ of species were represented by 19 genera with two genera of Charophyta (10.4\%) and 17 of Chlorophyta algae. Eight types of thallus structure were found with predominance of the lowly organized structure as a result of growing in an unstable environment. In the seasonal dynamics, the decrease in species richness with increasing altitude was revealed. Statistical analysis of relation between algal species richness and environmental variables suggests that altitude is a regulating factor of algal diversity development because the water temperature stimulates species richness, while altitude suppresses it. Therefore, mild summer and favorable spring seasons result in prolific vegetative growth of Chlorophyta and Charophyta algae species in the Swat River Basin habitats, but algal communities in the investigated area can be subjected to pollution impact. The results of this research will help in developing strategies for the conservation of aquatic ecosystems in Pakistan since the studied high-altitude areas of the Swat Basin can be seen as reference areas.
\end{abstract}

Keywords: Chlorophyta, Charophyta, species diversity, seasonal variations, Swat, Pakistan

РАЗНООБРАЗИЕ И СЕЗОННАЯ ДИНАМИКА СООБЩЕСТВ ЗЕЛЕНЫХ
(СНLОRОРНУТА RЕІСНЕNВАСН, 1828) И ХАРОФИТОВЫХ (СНАRОРНУТА
МIGULA, 1890) ВОДОРОСЛЕЙ В БАССЕЙНЕ РЕКИ СВАТ, ПАКИСТАН

Асгар Али ${ }^{1,2}$, Лал Бадшах ${ }^{2}$, София Баринова ${ }^{3 *}$

${ }^{1}$ Govt AKL Post Graduate College Maтma, Сват, Пакистан

${ }^{2}$ Кафедра ботаники, Университет Пешавара, Хайбер-Пахтунхва, Олд Джамруд Роуд, Пакистан, РК-25120

${ }^{3}$ Институт эволюиии, Университет Хайфы, Хайфа 3498838, Израиль

*E-mail:sophia@evo.haifa.ac.il 


\begin{abstract}
Аннотация. Растущее загрязнение воды и климатические изменения влияют на популяции водорослей и, в свою очередь, на региональные экосистемы. Данное исследование проведено с целью выявления флоры водорослей в пяти различных районах округа Сват, Хайбер-Пахтунхва, Пакистан, на высоте 972-2061 м над уровнем моря. Всего 105 таксонов водорослей из отделов Charophyta и Chlorophyta были впервые обнаружены в 200 образцах, собранных в течение 2007-2016 гг. Число видов Chlorophyta с 86 таксонами заметно преобладало, в отличие от Charophyta (19 таксонов). Показатели прозрачности воды и железа имеют положительную корреляцию с высотой, тогда как температура воды, рН, общая минерализация, аммиак, концентрация нитратов и цинк коррелируют с высотой местообитания отрицательно. Более 50 \% видов представлены 19 родами, в том числе двумя родами Charophyta (10,1\%) и 17 родами Chlorophyta. Обнаружено, что у восьми типов структуры таллома преобладает низкоорганизованная структура, что является результатом роста популяций водорослей в нестабильной среде. Выявлено снижение видового богатства с увеличением высоты в сезонной динамике. Статистический анализ взаимосвязи между видовым богатством водорослей и переменными среды показал, что высота является регулирующим фактором развития разнообразия водорослей, так как повышение температуры воды стимулирует, а увеличение высоты местообитания, наоборот, подавляет видовое богатство. Таким образом, мягкое лето и благоприятный весенний сезон приводят к активному вегетативному росту видов водорослей из отделов Chlorophyta и Charophyta в местообитаниях бассейна реки Сват; кроме того, выявлено, что сообщества водорослей в данном районе могут подвергаться воздействию загрязнения. Настоящее исследование может помочь при разработке стратегии по сохранению водных экосистем в Пакистане, поскольку изучены высокогорные участки бассейна крупной реки Сват, которые могут рассматриваться как фоновые.
\end{abstract}

Ключевые слова: Chlorophyta, Charophyta, видовое разнообразие, сезонная изменчивость, Сват, Пакистан

\section{INTRODUCTION}

The algal diversity research in the rivers of the southern Hindu Cush region is at its initial stage. In Pakistan, our knowledge of regional algal diversity is far from being exhaustive, whereas the algal communities of some rivers such as the Swat River and Kabul River up to its inflow to the Indus River have been studied better [1-8]. It is very important to reveal the distribution of different algal taxonomic and ecologic groups in the areas like Upper Indus Basin because the biodiversity of algal communities there was formed under natural climatic and anthropogenic impacts. Algal flora helps in assessment of aquatic ecosystems, their productivity and water quality [9]. Algal ecology enables proper understanding of aquatic habitats [10-12].

It is well known that freshwater algae are communities formed from members of different taxonomic divisions. Our previous study [1] revealed differences in sensitivity of algae from Bacillariophyta and other non-diatom species to climatic and anthropogenic impacts. While diatom algae usually make up more than a half of the community in rivers and streams and, thus, determine the response of the community as a whole, the Chlorophyta and Charophyta species are more sensitive to climatically related fluctuations of the environmental variables. Chlorophyta and Charophyta algae are different in their thallus organization that can be used also as a response of its structure to the environmental stress, because structure that is more complicated is typical of a more sustainable community. Species richness of the algae genera can also be very characteristic. That is, communities of undisturbed or protected water bodies are formed in the process of evolution with the advantage for diversifying individual taxa that are most adapted to this combination of climatic and environmental variables.

Seasonality of the algal community in closely related regions of southern Eurasia is studied in relation to climatic factors and altitude gradients [1]. Shrestha and Rai [13] identified 46 species of algae belonging to 34 genera from Rajrani Lake, Nepal. They also recorded seasonal variations among algal members. Soni and Thomas [14] reported 38 algal species from three sites in Gujrat, India. They studied interaction, dominance, and interdependence among various components of phytoplankton. Shams et al. [15] studied seasonal variations among algal members in Zayandeh-Rood Dam Lake, Isfahan, Iran. They reported 112 species of algae belonging to 52 genera. Asghar et al. [16] recorded 138 species of Chlorophycean members belonging to 25 families, from 10 different localities of District Swat. Sarim et al. [17] prepared a checklist of the algae from Sardaryab, Charsadda. Reshmi [18] identified 52 species of Chlorophyta from wetlands of Satna, Madhya Pradesh, India. Leghari et al. $[19,20]$ documented the algal flora of the Kunhar River, Pakistan, and Ertan 
and Morkoyunlu [21] reported 73 species of the algae from the Aksu stream, Isparta, Turkey. Thus, prior to this study, 149 species and infraspecies of the algae belonging to four taxonomic divisions were identified in the Swat River itself [1], where they demonstrated a clear response of the algal community structure to climatic and altitude gradients with an increasing role of Charophyta in contrary to chlorophytes species richness with increasing of altitude.

The aim of the present research was to reveal the species of Chlorophyta and Charophyta algae, studied for the first time in high mountain habitats of the Swat River Basin, and identify its community's response to the environmental factors gradients.

\section{MATERIALS AND METHODS}

\section{Description of the studied sites}

The Swat River is a river in Khyber-Pakhtunkhwa, Pakistan. Its source is in the Hindu Kush Mountains, from where it flows through the Kalam Valley passing from Tehsil Matta and Swat District. It then skirts Lower Dir District and flows through Malakand District to enter the Kabul River at Charsadda in the Peshawar valley and then follow to the Indus River. The Upper Swat consists of two main valleys, Sakhra and Rodingar, and many small tributaries, the sources of which are located in the Hindu Cush high mountains [22]. Most part of the Swat valley falls in a moist temperate zone characterized by cool winters and mild, pleasant summers. Water in the Swat River and its tributaries is used for agricultural needs.

\section{Sampling}

The sampling was conducted in the tributaries of the Swat River in all four seasons during the period of 2007-2016 from five monitoring sites: Saidu Sharif, Kanju, Khwaza Khela, Madyan, and Kalam (fig. 1). The samples of algae were collected by scraping and placed to polythene bags. The algal and water samples, taken concurrently, were transported to the lab in the icebox. Water transparency, dissolved oxygen, temperature, $\mathrm{pH}$, turbidity, total dissolved solids (TDS), and total suspended solids (TSS) were measured with the help of portable HANNA meters at the sampling point. As for ammonia, total alkalinity, nitrates, copper, iron, and zinc, they were determined in the laboratory of the Phytoecology of the Department of Botany, University of Peshawar by using standard techniques.

\section{Analysis of environmental variables}

GIS coordinates and altitude of sampling points were determined with GARMIN equipment. Water temperature was measured by thermometer; water $\mathrm{pH}$, turbidity, dissolved oxygen (DO) were measured with Portable HANNA Instruments selective equipment at the sampling site. Chemical variables were defined from the 1-liter water samples in the Laboratory of the Department of Botany, University of Peshawar, Pakistan using accepted methods of APHA [23].

\section{Preservation}

Each sample was taken in a polythene bag with some water from the locality, and later each of them was preserved in $3 \% \mathrm{FAA}$, i.e. formaldehyde solution, acetic acid and ethyl alcohol [24].

\section{Identification}

Algal samples were placed on a glass slide with a few drops of water and studied under BH-2 microscope. The objectives used were $10 \mathrm{X}$ and $20 \mathrm{X}$ with an eyepiece with ruled ocular. Identification was done according to the international handbooks [25-29]. The list of the revealed algae was updated with algaebase.org [30].

\section{Statistical methods}

Statistical programs Statistica 12.0 and JASP were used for calculation of algal species richness and environmental variables relationships. The network graphs and Pearson correlation matrix are produced by JASP based on the R Statistica package [31].

\section{RESULTS AND DISCUSSION}

\section{Environmental variables}

The averaged values of the environmental variables are presented in table 1 . The lowest water temperatures $\left(4.3^{\circ} \mathrm{C}\right)$ were recorded in Kalam. Temperature readings were reported as $5.7^{\circ} \mathrm{C}$ in Madyan, followed by Saidu Sharif $\left(6.7^{\circ} \mathrm{C}\right)$, Khwaza Khela $\left(8.7^{\circ} \mathrm{C}\right)$, and Kanju $\left(8.8^{\circ} \mathrm{C}\right)$. The lowest $\mathrm{pH}$ values $(6.9)$ were found at Madyan, and the highest $\mathrm{pH}$ (7.9) was documented at Saidu Sharif. Oxygen content was in an acceptable range at all monitoring sites. Dissolved oxygen was estimated as $9.6 \mathrm{mg} / \mathrm{L}$ at Madyan followed by $9.4 \mathrm{mg} / \mathrm{L}$ at Kalam, $9.3 \mathrm{mg} / \mathrm{L}$ at Saidu Sharif, $9.1 \mathrm{mg} / \mathrm{L}$ at Kanju, and $8.4 \mathrm{mg} / \mathrm{L}$ at Khwaza Khela. Ammonia nitrogen was found to be $0.02 \mathrm{ppm}$ at both Madyan and Kalam, while at Saidu Sharif and Khwaza Khela it equaled $0.05 \mathrm{ppm}$, and only $0.04 \mathrm{ppm}$ at Kanju. Total alkalinity was recorded as $121 \mathrm{ppm}$ at Saidu Sharif, $101 \mathrm{ppm}$ at Kanju, $74 \mathrm{ppm}$ at Khwaza Khela, 105 ppm at Madyan, and $85 \mathrm{ppm}$ at Kalam. Highest level of nitrates $(5.1 \mathrm{ppm})$ was documented at Kanju followed by $4.7 \mathrm{ppm}$ at Saidu Sharif, $2.3 \mathrm{ppm}$ at Madyan, and $1.8 \mathrm{ppm}$ at Kalam. Nitrate content was lowest at Khwaza Khela (1.4 ppm). Copper was below detection range at 


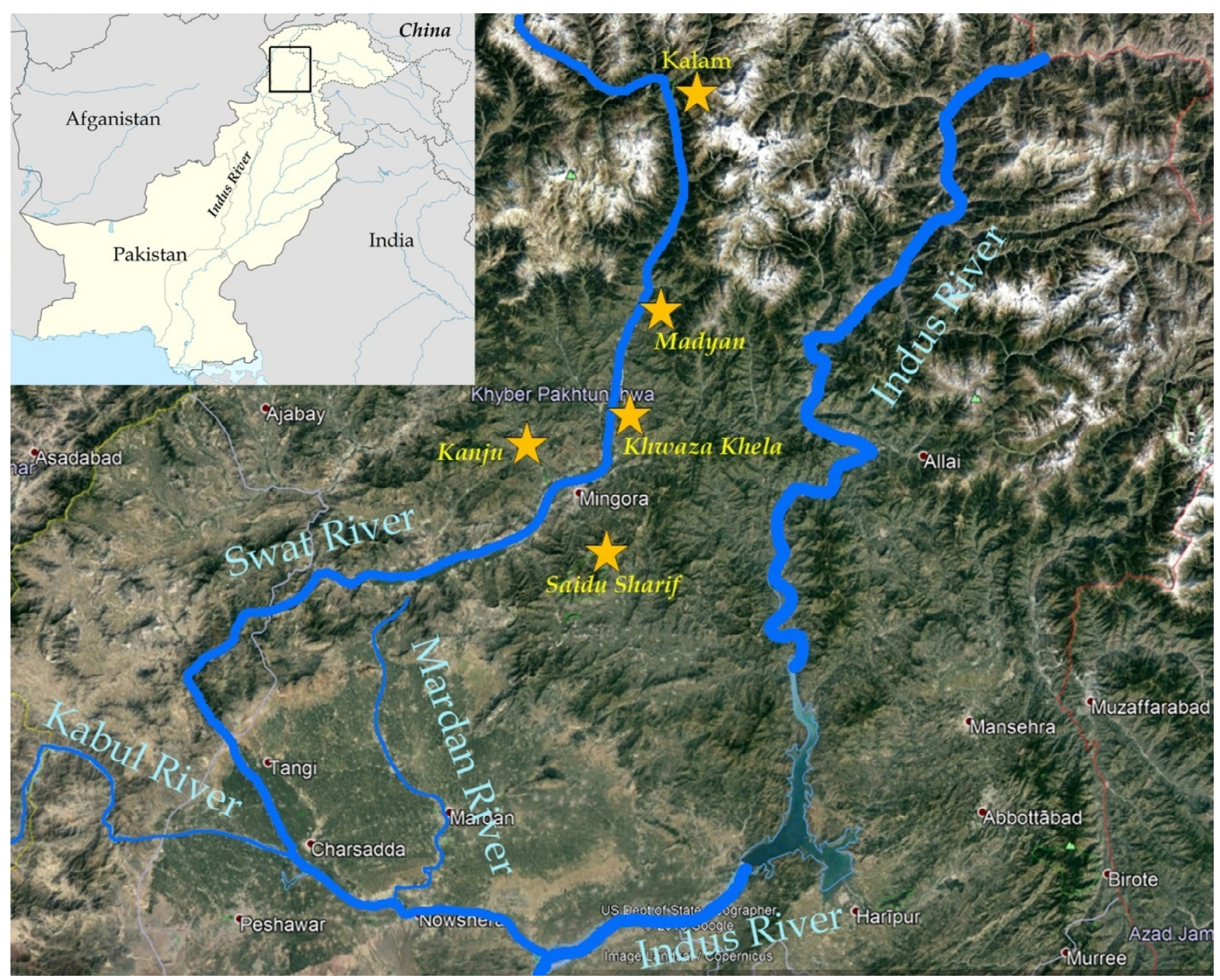

Fig. 1. Sampling sites in the Swat River Basin, orange stars

Рис. 1. Участки отбора проб в бассейне р. Сват, оранжевые звезды

Saidu Sharif and Kalam monitoring sites. The lowest copper level (0.001 ppm) was revealed at Khwaza Khela. Water samples from Kalam were found to have the highest iron level $(1.4 \mathrm{ppm})$ followed by 1.2 and $1.1 \mathrm{ppm}$ at Kanju and Madyan, respectively. The iron level was below detection range at Khwaza Khela. Highest value for zinc (3.6 ppm) was detected at Kanju, followed by Saidu Sharif (2.1 ppm). Zinc levels were below detection range at both Khwaza Khela and Kalam. Table 1 demonstrates synchronously changing variables of copper, ammonia, nitrates, and zinc with maximal values at site Kanju.

\section{Algal diversity}

Altogether, 105 taxa of Charophyta (19) and Chlorophyta (86) algae were revealed in 200 samples of algae collected from five sites in the Swat River Basin during 2007-2016 (table 2). Before analyzing the structure of the communities, the Willis curve was constructed (fig. 2), which is a criterion of representativeness of the identified species richness for floristic analysis and comparative floristics on algae [32]. It should be noted that distribution of species number in genera is close to the trend line; therefore, this species diversity can be analyzed as a continuum. So, Chlorophyta and Charophyta algae belong to 68 genera, saturation of which is represented in table 3. So, Cosmarium (Charophyta) was the leading genus, having 7 species $(6.67 \%)$, followed by Closterium with 4 species $(3.81 \%)$ also from Charophyta, and Chlamydomonas with 4 species from Chlorophyta. Oocystis, Coelastrum, Tetraëdron, and Ulothrix from Chlorophyta each had 3 species $(2.86 \%)$ while the next 17 genera were represented by 2 species each $(1.9 \%)$. The last 44 genera included 1 species each $(0.95 \%)$ while only four genera belonged to Charophyta. A checklist of recorded species is presented in table 2 . 
Table 1. Averaged environmental variables of five monitoring sites in the Swat River Basin in 2007-2016 with geographical position and altitude

Таблица 1. Средние значения параметров окружающей среды на пяти участках мониторинга в бассейне р. Сват в 2007-2016 гг. с указанием географического положения и высоты над уровнем моря

\begin{tabular}{|c|c|c|c|c|c|c|}
\hline $\begin{array}{l}\text { Parameters } \\
\text { Параметры }\end{array}$ & $\begin{array}{c}\text { Units } \\
\text { Ед. } \\
\text { измерения }\end{array}$ & $\begin{array}{c}\text { Saidu Sharif } \\
\text { Сайду- } \\
\text { Шариф }\end{array}$ & $\begin{array}{l}\text { Kanju } \\
\text { Канжу }\end{array}$ & $\begin{array}{c}\text { Khwaza Khela } \\
\text { Хваза Хела }\end{array}$ & $\begin{array}{l}\text { Madyan } \\
\text { Мадьян }\end{array}$ & $\begin{array}{l}\text { Kalam } \\
\text { Калам }\end{array}$ \\
\hline $\begin{array}{l}\text { Coordinate N } \\
\text { Координата с. ш. }\end{array}$ & $\begin{array}{c}\text { Grade } \\
\text { градус }\end{array}$ & $34^{\circ} 45^{\prime} 12^{\prime \prime}$ & $34^{\circ} 49^{\prime} 47^{\prime \prime}$ & $34^{\circ} 56^{\prime} 29^{\prime \prime}$ & $35^{\circ} 08^{\prime} 41^{\prime \prime}$ & $35^{\circ} 30^{\prime} 25^{\prime}$ \\
\hline $\begin{array}{l}\text { Coordinate E } \\
\text { Координата в. д. }\end{array}$ & $\begin{array}{l}\text { Grade } \\
\text { градус }\end{array}$ & $72^{\circ} 21^{\prime} 24^{\prime \prime}$ & $72^{\circ} 20^{\prime} 20^{\prime \prime}$ & $72^{\circ} 28^{\prime} 07^{\prime \prime}$ & $72^{\circ} 32^{\prime} 16^{\prime \prime}$ & $72^{\circ} 36^{\prime} 32^{\prime}$ \\
\hline $\begin{array}{l}\text { Altitude } \\
\text { Высота над уровнем моря }\end{array}$ & $\begin{array}{l}\text { м a.s.l. } \\
\text { м H.y.M. }\end{array}$ & 972 & 1,078 & 1,153 & 1,352 & 2,061 \\
\hline $\begin{array}{l}\text { Water surface temperature } \\
\text { Температура поверхности } \\
\text { воды }\end{array}$ & ${ }^{\circ} \mathrm{C}$ & 6.7 & 8.8 & 8.7 & 5.7 & 4.3 \\
\hline $\mathrm{pH}$ & - & 7.9 & 7.8 & 7.8 & 6.9 & 7.2 \\
\hline $\begin{array}{l}\text { TDS } \\
\text { Общая минерализация }\end{array}$ & $\begin{array}{l}\mathrm{ppm} \\
\mathrm{M \Gamma} / \text { Л }\end{array}$ & 81 & 87 & 78 & 52 & 55 \\
\hline $\begin{array}{l}\text { Turbidity } \\
\text { Мутность }\end{array}$ & $\begin{array}{l}\text { NTU } \\
\text { HEM }\end{array}$ & 5 & 4 & 2 & 1.5 & 1.4 \\
\hline $\begin{array}{l}\text { Transparency } \\
\text { Прозрачность }\end{array}$ & $\begin{array}{l}\mathrm{m} \\
\mathrm{M}\end{array}$ & 0.61 & 0.91 & 0.30 & 0.61 & 1.22 \\
\hline $\begin{array}{l}\text { DO } \\
\text { Растворенный кислород }\end{array}$ & $\begin{array}{c}\mathrm{mg} / \mathrm{L} \\
\mathrm{Mг} / \mathrm{J}\end{array}$ & 9.3 & 9.1 & 8.4 & 9.6 & 9.4 \\
\hline $\begin{array}{l}\text { Ammonia } \\
\text { Аммиак }\end{array}$ & $\begin{array}{l}\mathrm{ppm} \\
\mathrm{Mг} / \pi\end{array}$ & 0.05 & 0.04 & 0.05 & 0.02 & 0.02 \\
\hline $\begin{array}{l}\text { Total alkalinity } \\
\text { Общая щелочность }\end{array}$ & $\begin{array}{l}\mathrm{ppm} \\
\mathrm{M \Gamma} / л\end{array}$ & 121 & 101 & 74 & 105 & 85 \\
\hline $\begin{array}{l}\text { Nitrates } \\
\text { Нитраты }\end{array}$ & $\begin{array}{l}\mathrm{ppm} \\
\mathrm{M \Gamma} / \pi\end{array}$ & 4.7 & 5.1 & 1.4 & 2.3 & 1.8 \\
\hline $\begin{array}{l}\text { Copper } \\
\text { Медь }\end{array}$ & $\begin{array}{l}\mathrm{ppm} \\
\mathrm{M \Gamma} / л\end{array}$ & 0 & 0.07 & 0.001 & 0.003 & 0 \\
\hline $\begin{array}{l}\text { Iron } \\
\text { Железо }\end{array}$ & $\begin{array}{l}\mathrm{ppm} \\
\mathrm{M \Gamma} / \pi\end{array}$ & 0.3 & 1.2 & 0 & 1.1 & 1.4 \\
\hline $\begin{array}{l}\text { Zinc } \\
\text { Цинк }\end{array}$ & $\begin{array}{l}\mathrm{ppm} \\
\mathrm{MГ} / л\end{array}$ & 2.1 & 3.6 & 0 & 0.5 & 0 \\
\hline
\end{tabular}

Variation among algal diversity at different monitoring sites can be attributed to different physico-chemical attributes [20]. The head part of the studied diversity that included more than $50 \%$ of species is shadowed in table 3. It contains only two Charophyta genera with 11 species $(10.1 \%)$ but the rest ones are Chlorophyta algae.

\section{Thallus structure}

Eight different types of thallus organisation were identified among the collected algal species (table 4). Colonial members were most abundant, represented by 17 genera (34\%). These were followed by unicellular forms (12 genera, $24 \%$ ), unbranched ( 8 genera, $16 \%$ ) and branched filamentous forms (5 genera, $10 \%$ ).
Irregular thalli were found in 4 genera $(8 \%)$ and two genera had pseudo-filamentous thalli (4\%). Hydrodictyon reticulatum and Coleochaete orbicularis were the only species with mesh-like and heterotrichous thalli, respectively. Distribution according to the type of thallus structure allowed to assume that the revealed algae represent mostly lowly organized structure and, thus, developed in an unstable environment. Prevalence of colonial and unicellular forms here is similar to the results reported by Haroon et al. [33] where it is mentioned that domestic waste in this region contains numerous toxic substances, which gives evidence of the algal species richness. 


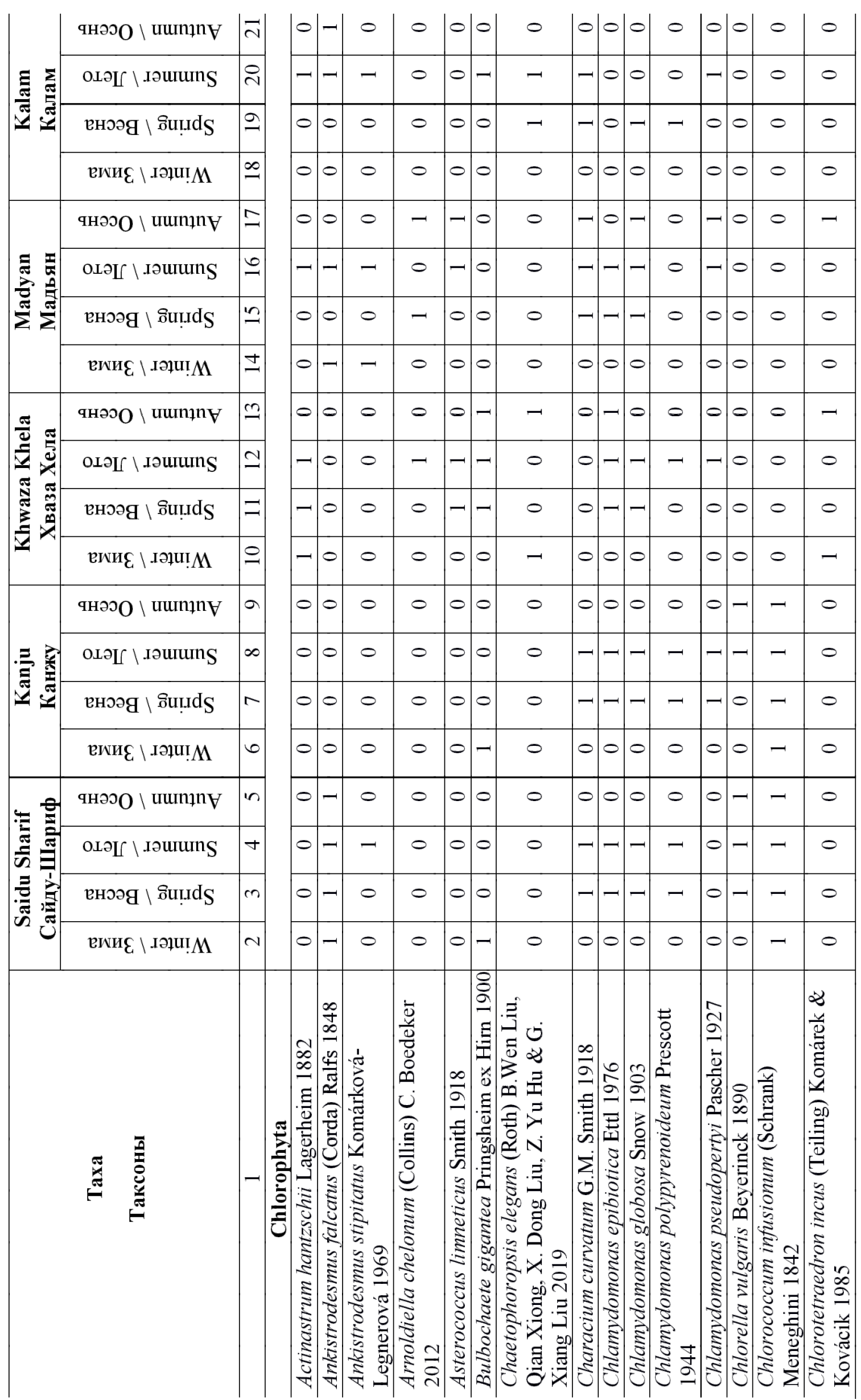




\begin{tabular}{|c|c|c|c|c|c|c|c|c|c|c|c|c|c|c|c|c|c|c|c|c|c|}
\hline $\bar{\sim}$ & 0 & 1010 & 010 & 010 & 10 & 10 & 0 & 0 & 0 & 0 & 0 & 0 & 0 & 0 & & & 0 & 0 & 0 & 0 & 0 \\
\hline & 0 & $-1-$ & -1 & 0.0 & 0 & - & 0 & & 0 & - & 0 & 0 & - & 0 & & 0 & 0 & - & 0 & 0 & \\
\hline
\end{tabular}

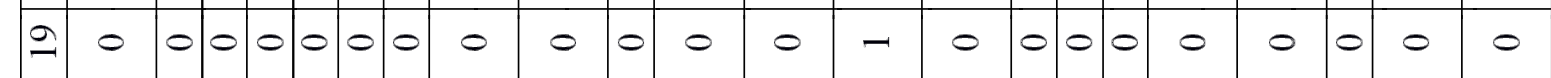

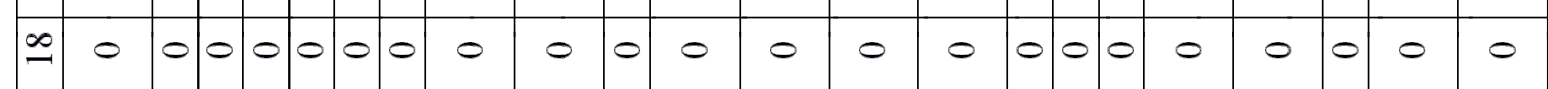
I 0 -

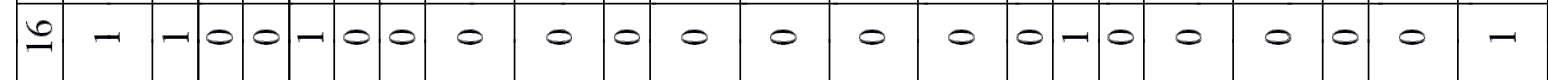

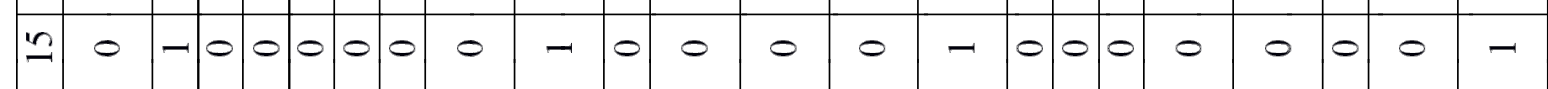

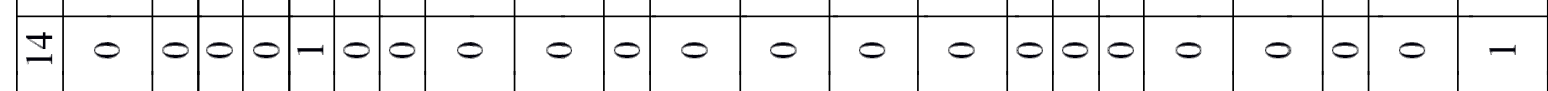

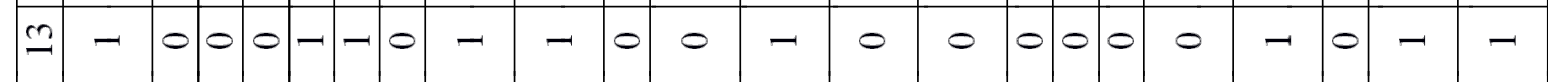

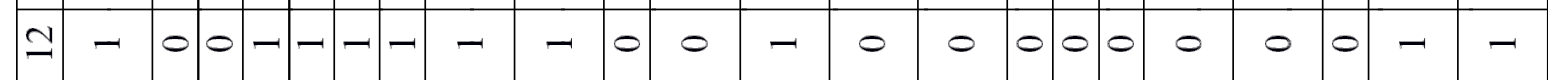

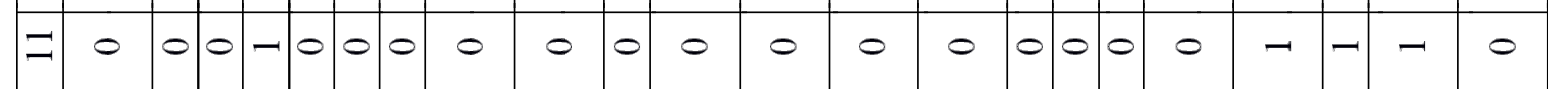

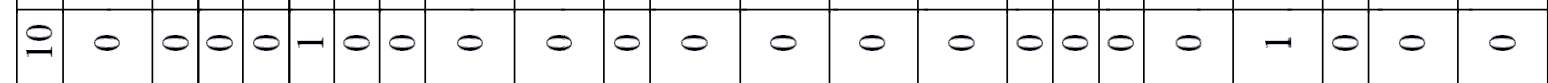

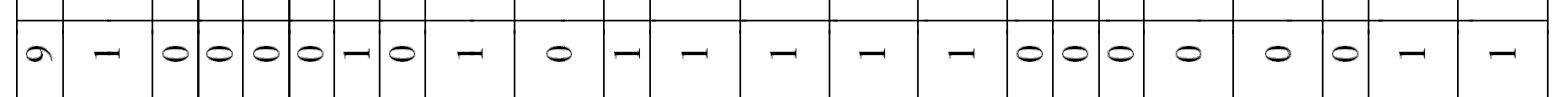

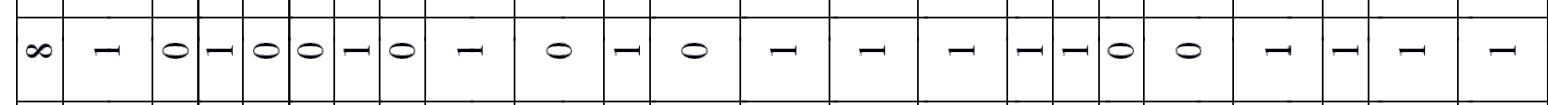

\begin{tabular}{rlllllllllllllllll|l|l|l|l|l|l|}
\hline$r$ & - & 0 & 0 & 0 & 0 & - & 0 & 0 & 0 & 0 & 0 & - & 0 & 0 & - & - & 0 & 0 & -1 & -1 & 0 & -1 \\
\hline
\end{tabular}

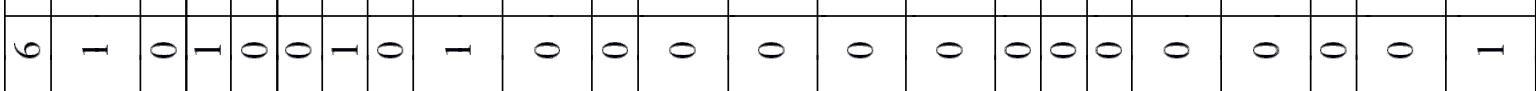

\begin{tabular}{|c|c|c|c|c|c|c|c|c|c|c|c|c|c|c|c|c|c|c|c|c|c|}
\hline & & & & & & & & & & & & & & & & & & & & & \\
\hline in & - & $-1-$ & -10 & 0 & $-1-$ & & - & 0 & 0 & 0 & - & - & 0 & 0 & 0 & 0 & 0 & 0 & 0 & 0 & - \\
\hline
\end{tabular}

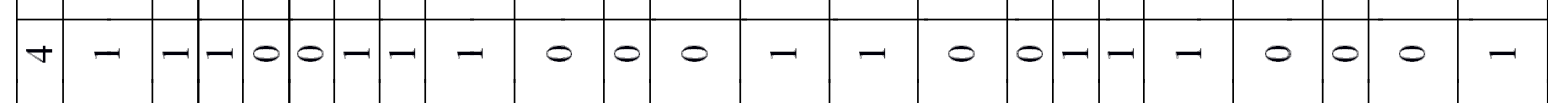

\begin{tabular}{|c|c|c|c|c|c|c|c|c|c|c|c|c|c|c|c|c|c|c|c|c|c|c|}
\hline m & - & 0 & 0 & 0 & 0 & -1 & - & 0 & 0 & 0 & 0 & - & 0 & 0 & 0 & - & -1 & - & 0 & 0 & 0 & - \\
\hline$N$ & - & - & - & - & - & -1 & - & - & 0 & 0 & 0 & 0 & 0 & 0 & 0 & 0 & 0 & 0 & 0 & 10 & 0 & - \\
\hline
\end{tabular}

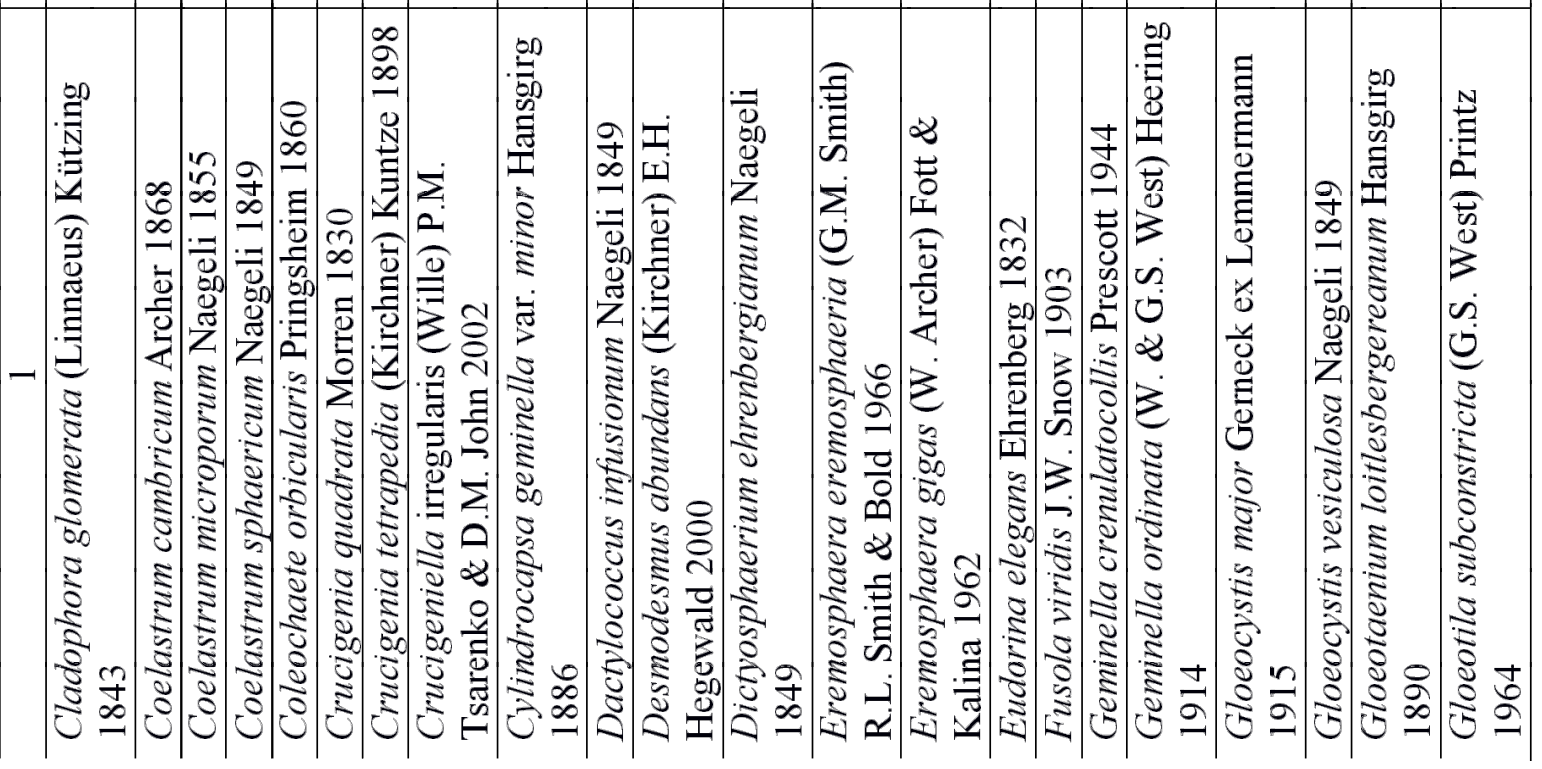




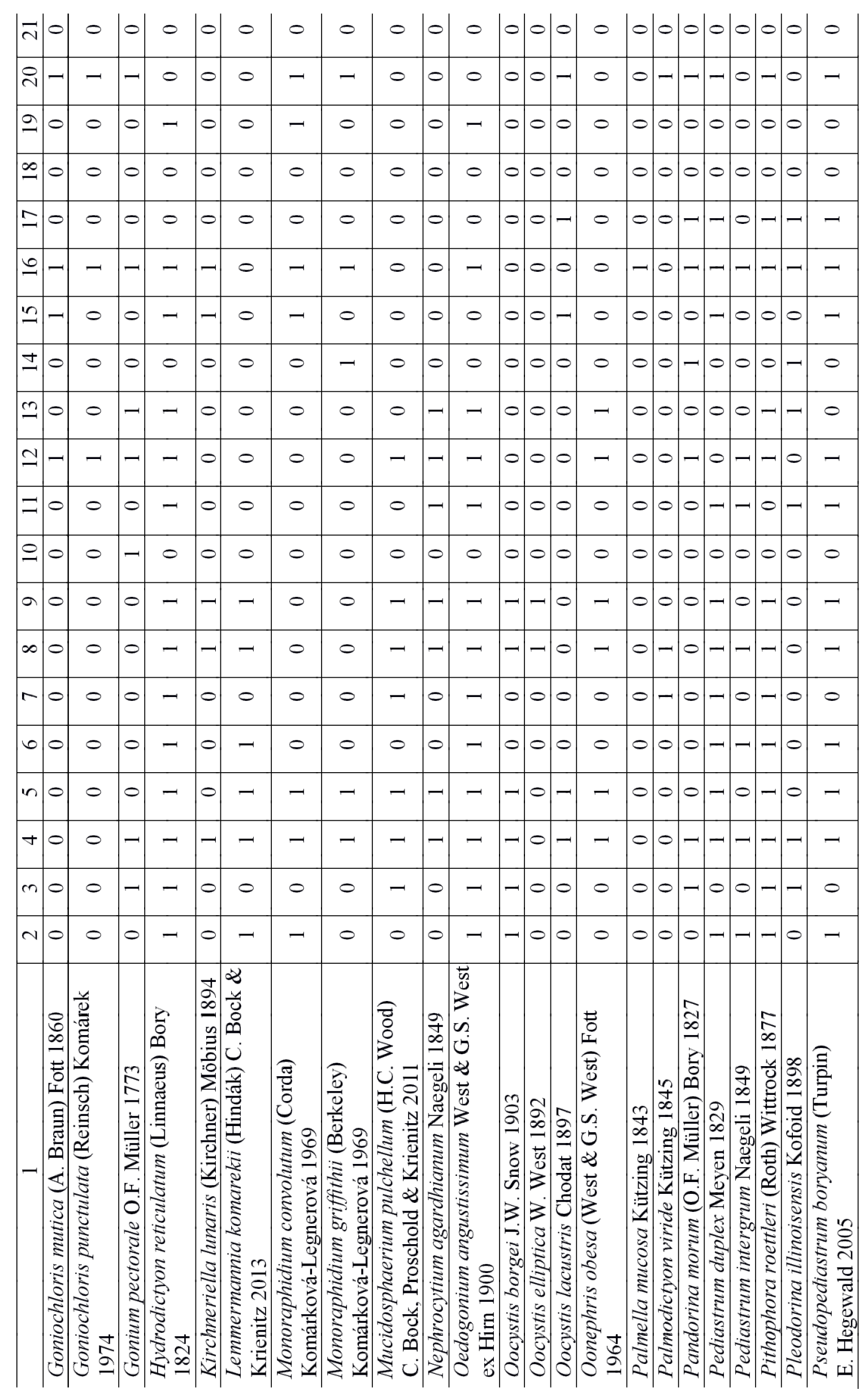




\begin{tabular}{|c|c|c|c|c|c|c|c|c|c|c|c|c|c|c|c|c|c|c|c|c|c|c|c|}
\hline 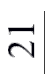 & 0 & 0 & 0 & 0 & 0 & 0 & 0 & 0 & 0 & 0 & 0 & 0 & 0 & 0 & 0 & & 010 & 10 & 0 & 0 & 0 & 0.0 & 0 \\
\hline & 0 & 0 & 0 & 0 & 0 & -1 & 0 & - & 0 & - & -1 & 0 & 0 & 0 & 0 & 0 & 010 & 0 & 0 & 0. & -1. & -10 & 0 \\
\hline & 0 & 0 & 0 & 0 & 0 & 0 & 0 & 0 & 0 & 0 & 0 & 0 & 0 & 0 & 0 & 00 & 010 & 0 & 0 & 0. & -1. & -10 & 0 \\
\hline & 0 & 0 & 0 & 0 & 0 & 0 & 10 & 0 & 0 & 0 & 0 & 0 & 0 & 0 & 0 & 00 & 0 & 10 & 0 & 0 & 0 & 010 & 0 \\
\hline & 0 & 0 & 0 & 0 & 0 & - & e & $=$ & 0 & 0 & -1 & 0 & 0 & 0 & - & $0=$ & 0 & 10 & 0 & -1 & -1 & -10 & 0 \\
\hline & - & 00 & 0 & 0 & -1 & -1 & 10 & 0 & 0 & - & -1 & 0 & 0 & -1 & - & 00 & 010 & - & 0 & - & -7. & -10 & 0 \\
\hline & 0 & 00 & 0 & 0 & 0 & - & 10 & - & 0 & - & 0 & - & 0 & -1 & 0 & 00 & 010 & 10 & 0 & 0 & 0. & 0.0 & 0 \\
\hline & - & 0 & 0 & 0 & -1 & 0 & 0 & 0 & 0 & 0 & 0 & 0 & 0 & 0 & - & 00 & 010 & - & 0 & 0. & -0 & 0.0 & 0 \\
\hline & 0 & 0 & 0 & 0 & 0 & 0 & 0 & 0 & 0 & 0 & 0 & 0 & 0 & 0 & 0 & -- & $-1=$ & 0 & 0 & -1 & - & --1 & 0 \\
\hline & - & 0 & 0 & -1 & -1 & - & 0 & - & 0 & 0 & 0 & -1 & 0 & 0 & - & -- & -10 & - & 0 & - & - & --1 & - \\
\hline & - & 0 & 0 & -1 & 0 & 0 & 0 & 0 & 0 & 0 & 0 & - & 0 & 0 & 0 & -- & -10 & - & - & 0. & -1. & -10 & - \\
\hline$\Xi$ & 0 & 0 & 0 & 0 & 0 & 0 & 0 & 0 & 0 & 0 & 0 & 0 & 0 & 0 & 0 & 00 & 00 & 0 & 0 & 0. & -1. & -0 & 0 \\
\hline & 0 & -1. & -1 & -1 & 0 & 0 & 0 & - & 0 & - & -1 & 0 & - & -1 & 0 & $0-1$ & -10 & 10 & - & -1 & 0 & $0:-1$ & - \\
\hline & - & -1 & - & -1 & - & - & 0 & & - & - & -1 & 0 & - & -1 & - & -- & $-1-$ & 10 & - & - & -7. & -10 & - \\
\hline 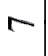 & - & 0 & 0 & -1 & - & -1 & 10 & & 0 & 0 & 0 & 0 & 0 & -1 & - & -0 & 010 & - & - & - & -1 & -10 & 0 \\
\hline 6 & 0 & 0. & -1 & 0 & 0 & 0 & 0 & - & 0 & 0 & 0 & 0 & 0 & -1 & 0 & 00 & 00 & - & -1 & - & 0 & 0.0 & 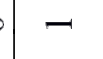 \\
\hline$r$ & 0 & 0. & - & 0 & 0 & 0 & - & - & 0 & 0 & 0 & 0 & - & -1 & 0 & 00 & 010 & 10 & - & - & 0 & 00 & 0 \\
\hline$\checkmark$ & 0 & 0. & - & -1 & - & $c$ & 0 & & 0 & 0 & 0 & 0 & 0 & -1 & - & 00 & 010 & 0 & - & -1 & 0 & -10 & - \\
\hline & 0 & 010 & 0 & -1 & -1 & 0 & 0 & & 0 & 0 & 0 & 0 & 0 & -1 & - & 0 & 00 & - & - & - & -1. & -10 & 0 \\
\hline 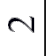 & 0 & 0 & - & 0 & 0 & 0 & 0 & & 0 & 0 & 0 & 0 & 0 & -1 & 0 & 00 & 010 & - & - & - & 0 & 0.0 & - \\
\hline & 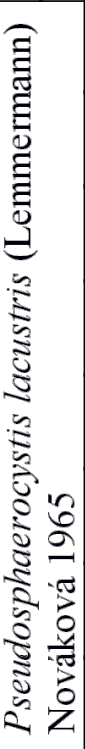 & 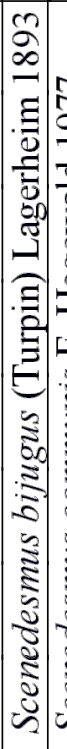 & 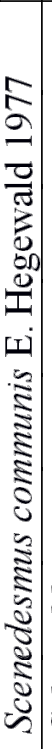 & 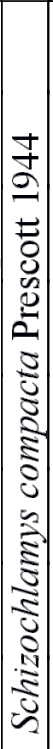 & & 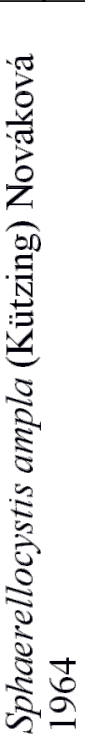 & 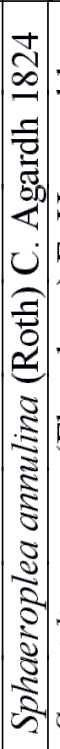 & 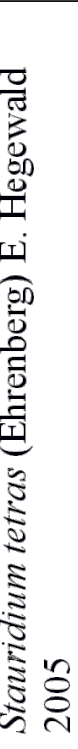 & 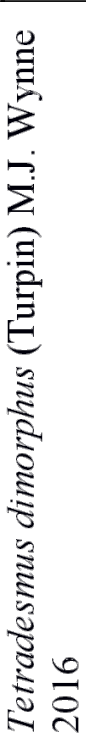 & 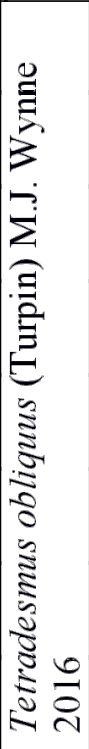 & 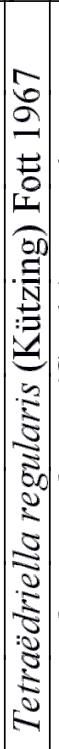 & 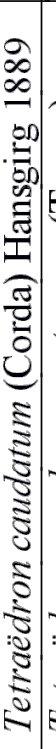 & 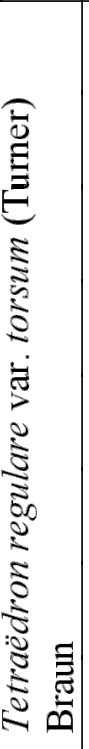 & 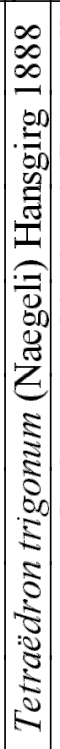 & 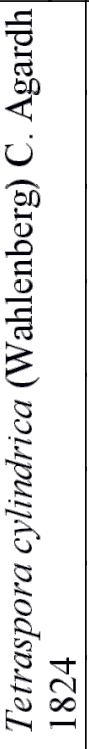 & 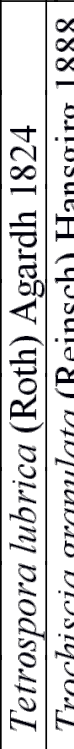 & 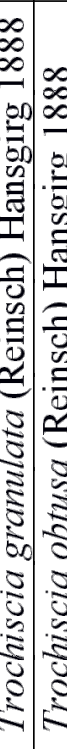 & 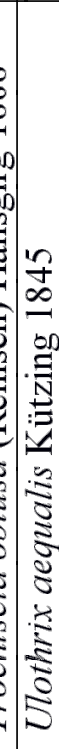 & 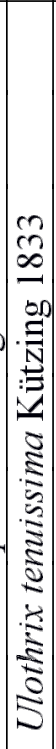 & 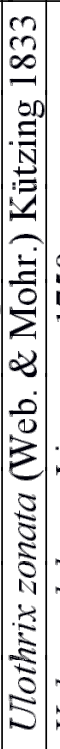 & 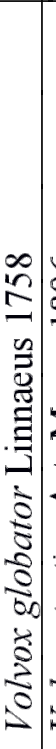 & 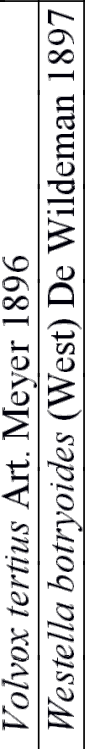 & 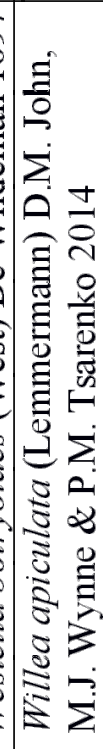 \\
\hline
\end{tabular}




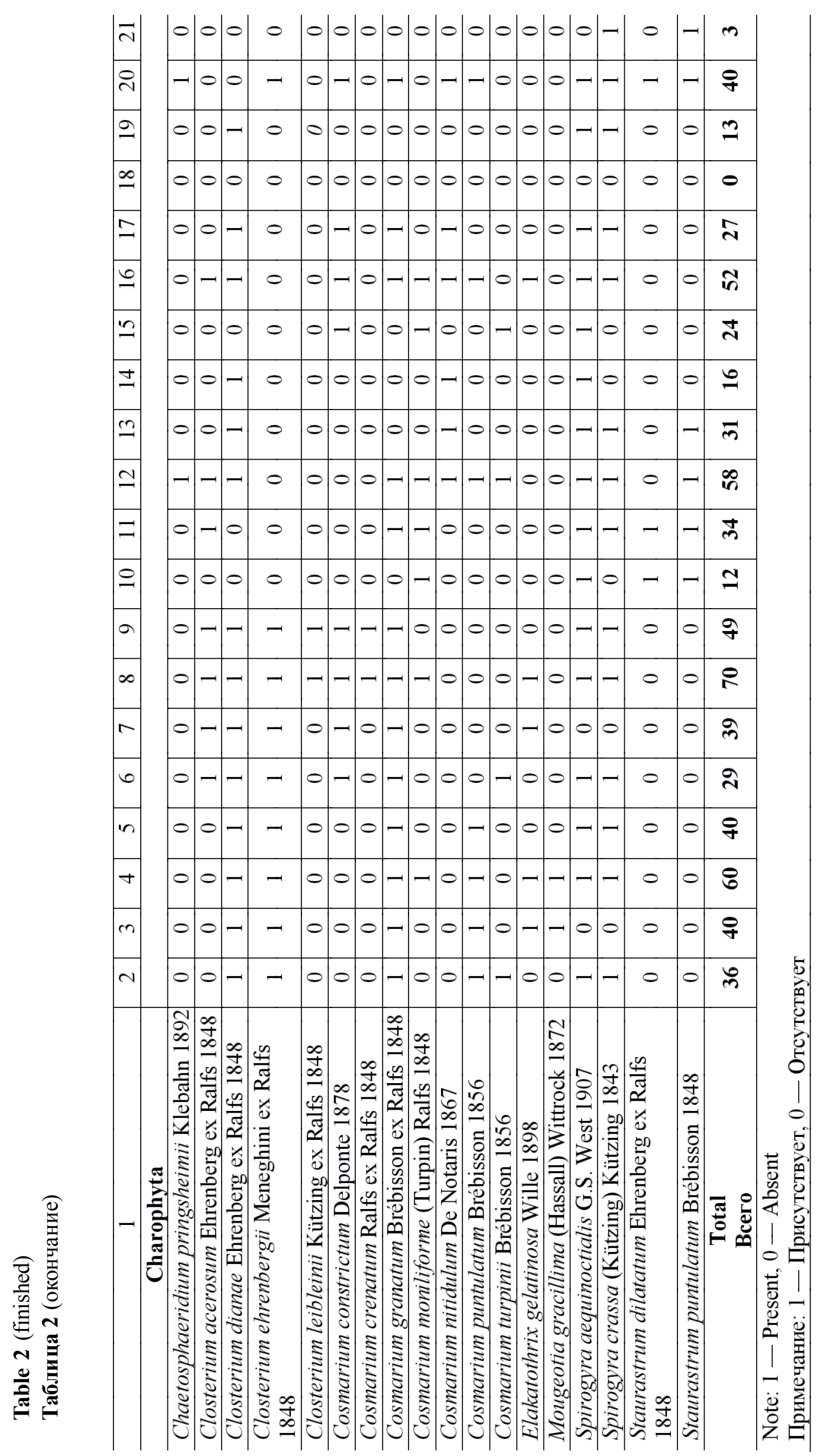




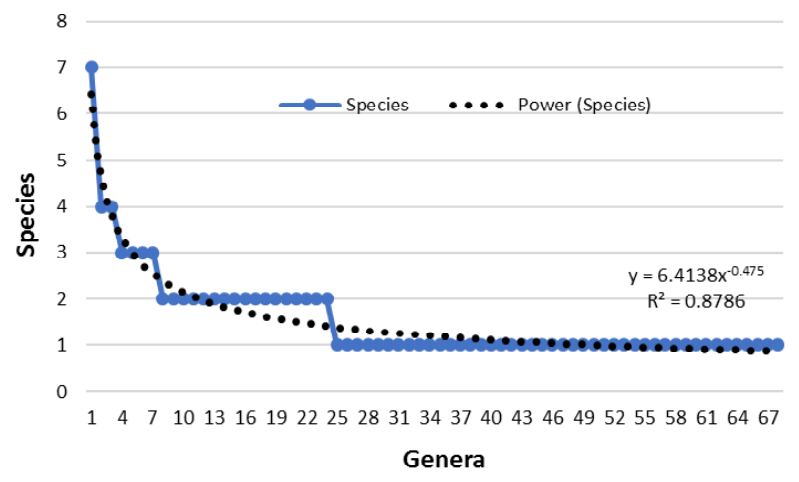

Fig. 2. Distribution of algal species over genera (Willis curve) in the Swat River Basin in 20072016

Рис. 2. Распределение видов водорослей по родам (кривая Виллиса) в бассейне р. Сват в 2007-2016 гг.

\section{Seasonal variations}

Seasonal variations of species richness are presented in table 5 and fig. 3. Highest species richness was observed during summer and spring seasons. At the lower site Saidu Sharif, 60 algal species were collected during summer followed by 40 species both in spring and autumn, whereas only 36 species were sampled during the winter season. At Kanju site, 70 algal species were documented in the summer season followed by

Table 3. Species saturation of genera of the Swat River valley algal communities with percentage of presenting. Charophyta genera are marked bold. $50 \%$ of species are shadowed

Таблица 3. Видовая насыщенность родов в водорослевых сообществах долины р. Сват с процентной долей представленности. Роды Charophyta выделены жирным шрифтом. Серым подсвечены виды, составляющие в сумме 50 \% от общего числа

\begin{tabular}{l|c|c}
\hline \multicolumn{1}{c|}{$\begin{array}{l}\text { Genera } \\
\text { Роды }\end{array}$} & $\begin{array}{c}\text { Species } \\
\text { Виды }\end{array}$ & $\begin{array}{c}\text { Percent of total } \\
\text { species, \% } \\
\text { Доля от } \\
\text { общего числа } \\
\text { видов, \% }\end{array}$ \\
\hline \multicolumn{1}{c|}{1} & 2 & 3 \\
\hline Cosmarium & $\mathbf{7}$ & $\mathbf{6 . 6 7}$ \\
\hline Chlamydomonas & 4 & 3.81 \\
\hline Closterium & $\mathbf{4}$ & $\mathbf{3 . 8 1}$ \\
\hline Coelastrum & 3 & 2.86 \\
\hline Oocystis & 3 & 2.86 \\
\hline Tetraëdron & 3 & 2.86 \\
\hline Ulothrix & 3 & 2.86 \\
\hline Pediastrum & 2 & 1.90 \\
\hline Ankistrodesmus & 2 & 1.90 \\
\hline
\end{tabular}

\begin{tabular}{|c|c|c|}
\hline 1 & 2 & 3 \\
\hline Crucigenia & 2 & 1.90 \\
\hline Eremosphaera & 2 & 1.90 \\
\hline Geminella & 2 & 1.90 \\
\hline Gloeocystis & 2 & 1.90 \\
\hline Goniochloris & 2 & 1.90 \\
\hline Monoraphidium & 2 & 1.90 \\
\hline Scenedesmus & 2 & 1.90 \\
\hline Schizochlamys & 2 & 1.90 \\
\hline Tetradesmus & 2 & 1.90 \\
\hline Tetraspora & 2 & 1.90 \\
\hline Trochiscia & 2 & 1.90 \\
\hline Volvox & 2 & 1.90 \\
\hline Willea & 2 & 1.90 \\
\hline Spirogyra & 2 & 1.90 \\
\hline Staurastrum & 2 & 1.90 \\
\hline Actinastrum & 1 & 0.95 \\
\hline Arnoldiella & 1 & 0.95 \\
\hline Asterococcus & 1 & 0.95 \\
\hline Bulbochaete & 1 & 0.95 \\
\hline Chaetophoropsis & 1 & 0.95 \\
\hline Characium & 1 & 0.95 \\
\hline Chlorella & 1 & 0.95 \\
\hline Chlorococcum & 1 & 0.95 \\
\hline Chloroidium & 1 & 0.95 \\
\hline Chlorotetraedron & 1 & 0.95 \\
\hline Cladophora & 1 & 0.95 \\
\hline Coleochaete & 1 & 0.95 \\
\hline Crucigeniella & 1 & 0.95 \\
\hline Cylindrocapsa & 1 & 0.95 \\
\hline Dactylococcus & 1 & 0.95 \\
\hline Desmodesmus & 1 & 0.95 \\
\hline Dictyosphaerium & 1 & 0.95 \\
\hline Eudorina & 1 & 0.95 \\
\hline Fusola viridis & 1 & 0.95 \\
\hline Gloeotaenium & 1 & 0.95 \\
\hline Gloeotila & 1 & 0.95 \\
\hline Gonium & 1 & 0.95 \\
\hline Hydrodictyon & 1 & 0.95 \\
\hline Kirchneriella & 1 & 0.95 \\
\hline Lemmermannia & 1 & 0.95 \\
\hline Mucidosphaerium & 1 & 0.95 \\
\hline Nephrocytium & 1 & 0.95 \\
\hline Oedogonium & 1 & 0.95 \\
\hline Oonephris & 1 & 0.95 \\
\hline Palmella & 1 & 0.95 \\
\hline Palmodictyon & 1 & 0.95 \\
\hline Pandorina & 1 & 0.95 \\
\hline Pithophora & 1 & 0.95 \\
\hline Pleodorina & 1 & 0.95 \\
\hline Pseudopediastrum & 1 & 0.95 \\
\hline Pseudosphaerocystis & 1 & 0.95 \\
\hline Sphaeroplea & 1 & 0.95 \\
\hline
\end{tabular}


Table 3 (finished)

Таблица 3 (окончание)

\begin{tabular}{l|l|l}
\hline \multicolumn{1}{c|}{1} & 2 & 3 \\
\hline Stauridium tetras & 1 & 0.95 \\
\hline Tetraëdriella & 1 & 0.95 \\
\hline Westella & 1 & 0.95 \\
\hline Chaetosphaeridium & $\mathbf{1}$ & $\mathbf{0 . 9 5}$ \\
\hline Elakatothrix & $\mathbf{1}$ & $\mathbf{0 . 9 5}$ \\
\hline Mougeotia & $\mathbf{1}$ & $\mathbf{0 . 9 5}$ \\
\hline Zygnema & $\mathbf{1}$ & $\mathbf{0 . 9 5}$ \\
\hline
\end{tabular}

49 species in autumn, 40 species in spring and 29 in winter. At Khwaza Khela site, 58 species were recorded in the summer season, 34 in spring, 31 in autumn, and only 12 species in the winter season. At Madyan monitoring site, 52 species were identified in summer, 27 in autumn, 24 in spring and 16 in the winter season. At the uppermost site Kalam, where temperatures fall below freezing point during winters, the highest number of species appeared in summer (40), followed by 13 in spring and 3 in autumn. This distribution demonstrated that the summer season is more favorable for algal community growth. Highest species richness was recorded during the summer season, which is in line with findings of Barinova et al. [1] for the Swat River itself, as well as Solak et al. [34] and Shams et al. [15].
The trend line for this distribution shows (fig. 3) the decrease in total species richness as well as Chlorophyta and Charophyta species number with the altitude of a site. It can be explained by the fact that altitude is a strong regulating factor for algal community growth in the Swat River Basin.

As the next step of the analysis, Pearson correlation coefficient for environmental variables and algae species richness was calculated. The calculation results

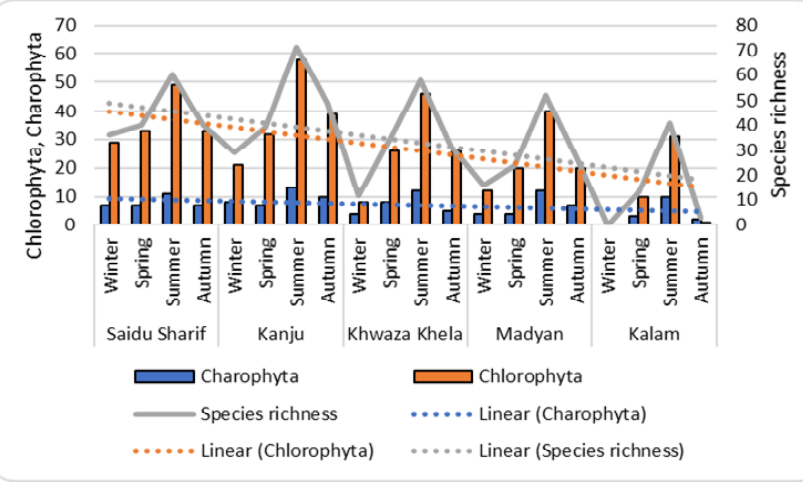

Fig. 3. Seasonal variations of species richness of algal communities in the Swat River valley with trend lines (dotted)

Рис. 3. Сезонные изменения видового богатства в водорослевых сообществах долины р. Сват. Пунктирные линии - линии тренда

Table 4. Number of genera and their distribution by types of thallus organization with percentage of total species richness of algal communities in the Swat River Basin

Таблица 4. Число родов и их распределение по типам организации таллома с процентной долей от общего видового богатства водорослевых сообществ в бассейне р. Сват

\begin{tabular}{|c|c|c|c|}
\hline $\begin{array}{c}\text { Order } \\
\text { Порядковое место }\end{array}$ & $\begin{array}{l}\text { Type of organization of thallus } \\
\text { Тип организации таллома }\end{array}$ & $\begin{array}{l}\text { No of genera } \\
\text { Число родов }\end{array}$ & $\begin{array}{l}\text { Percent of species, \% } \\
\text { Процент видов, \% }\end{array}$ \\
\hline 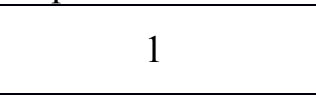 & $\begin{array}{l}\text { Colonial } \\
\text { Колониальный }\end{array}$ & 17 & 34.00 \\
\hline 2 & $\begin{array}{l}\text { Unicellular } \\
\text { Одноклеточный }\end{array}$ & 12 & 24.00 \\
\hline 3 & $\begin{array}{l}\text { Unbranched filamentous } \\
\text { Неразветвленный нитчатый }\end{array}$ & 8 & 16.00 \\
\hline 4 & $\begin{array}{l}\text { Branched filamentous } \\
\text { Разветвленный нитчатый }\end{array}$ & 5 & 10.00 \\
\hline 5 & $\begin{array}{l}\text { Irregular } \\
\text { Неправильный }\end{array}$ & 4 & 8.00 \\
\hline 6 & $\begin{array}{l}\text { Pseudofilamentous } \\
\text { Псевдонитчатый }\end{array}$ & 2 & 4.00 \\
\hline 7 & $\begin{array}{l}\text { Heterotrichous } \\
\text { Гетеротрихальный } \\
\text { (разнонитчатый) }\end{array}$ & 1 & 2.00 \\
\hline 8 & $\begin{array}{l}\text { Mesh-like } \\
\text { Сетчатый }\end{array}$ & 1 & 2.00 \\
\hline
\end{tabular}


Table 5. Seasonal variations of species richness in the Swat River valley algal communities

Таблица 5. Сезонные изменения видового богатства в водорослевых сообществах долины р. Сват

\begin{tabular}{|c|c|c|c|c|}
\hline $\begin{array}{c}\text { Site } \\
\text { Участок }\end{array}$ & $\begin{array}{c}\text { Season } \\
\text { Сезон }\end{array}$ & Charophyta & Chlorophyta & $\begin{array}{l}\text { Total } \\
\text { Всего }\end{array}$ \\
\hline \multirow{4}{*}{$\begin{array}{c}\text { Saidu } \\
\text { Sharif } \\
\text { Сайду- } \\
\text { Шариф }\end{array}$} & $\begin{array}{c}\text { Winter } \\
\text { Зима }\end{array}$ & 7 & 29 & 36 \\
\hline & $\begin{array}{l}\text { Spring } \\
\text { Весна }\end{array}$ & 7 & 34 & 40 \\
\hline & $\begin{array}{c}\text { Summer } \\
\text { Лето }\end{array}$ & 10 & 50 & 60 \\
\hline & $\begin{array}{c}\text { Autumn } \\
\text { Осень }\end{array}$ & 7 & 33 & 40 \\
\hline \multirow{4}{*}{$\begin{array}{l}\text { Kanju } \\
\text { Канжу }\end{array}$} & $\begin{array}{c}\text { Winter } \\
\text { Зима } \\
\end{array}$ & 8 & 21 & 29 \\
\hline & $\begin{array}{l}\text { Spring } \\
\text { Весна }\end{array}$ & 6 & 33 & 39 \\
\hline & $\begin{array}{c}\text { Summer } \\
\text { Лето }\end{array}$ & 12 & 58 & 70 \\
\hline & $\begin{array}{c}\text { Autumn } \\
\text { Осень }\end{array}$ & 10 & 39 & 49 \\
\hline \multirow{4}{*}{$\begin{array}{c}\text { Khwaza } \\
\text { Khela } \\
\text { Хваза } \\
\text { Хела }\end{array}$} & $\begin{array}{c}\text { Winter } \\
\text { Зима }\end{array}$ & 4 & 8 & 12 \\
\hline & $\begin{array}{l}\text { Spring } \\
\text { Весна }\end{array}$ & 8 & 26 & 34 \\
\hline & $\begin{array}{c}\text { Summer } \\
\text { Лето }\end{array}$ & 12 & 46 & 58 \\
\hline & $\begin{array}{c}\text { Autumn } \\
\text { Осень }\end{array}$ & 5 & 26 & 31 \\
\hline \multirow{4}{*}{$\begin{array}{l}\text { Madyan } \\
\text { Мадьян }\end{array}$} & $\begin{array}{c}\text { Winter } \\
\text { Зима } \\
\end{array}$ & 4 & 12 & 16 \\
\hline & $\begin{array}{l}\text { Spring } \\
\text { Весна } \\
\end{array}$ & 4 & 20 & 24 \\
\hline & $\begin{array}{c}\text { Summer } \\
\text { Лето }\end{array}$ & 11 & 41 & 52 \\
\hline & $\begin{array}{c}\text { Autumn } \\
\text { Осень }\end{array}$ & 7 & 20 & 27 \\
\hline \multirow{4}{*}{$\begin{array}{l}\text { Kalam } \\
\text { Калам }\end{array}$} & $\begin{array}{c}\text { Winter } \\
\text { Зима }\end{array}$ & 0 & 0 & 0 \\
\hline & $\begin{array}{l}\text { Spring } \\
\text { Весна } \\
\end{array}$ & 3 & 10 & 13 \\
\hline & $\begin{array}{c}\text { Summer } \\
\text { Лето }\end{array}$ & 10 & 30 & 40 \\
\hline & $\begin{array}{c}\text { Autumn } \\
\text { Осень }\end{array}$ & 2 & 1 & 3 \\
\hline
\end{tabular}

show (table 6) strong positive correlation between ammonia and water $\mathrm{pH}$, ammonia and TDS, turbidity and nitrates, and nitrates and zinc. All of these factors are related to anthropogenic pollution. Total species richness has a positive correlation with temperature and negative one with the site altitude. Chlorophyta species number was defined out of total species richness and stimulated by TDS of water. So, algal species richness is an indicator of aquatic pollution in the studied waterbodies of this region $[6,7]$.

An attempt was made to reveal the correlation of individual species richness in-season community with the help of a new approach at JASP statistical program. Fig. 4 presents results for the site grouping in which two community cores can be seen. Safe site relation, the first core combines communities from Saudi Sharif and Kanju sites that are close to each other and have similar climatic conditions. The second core combines communities from three other sites placed in the upper altitude.

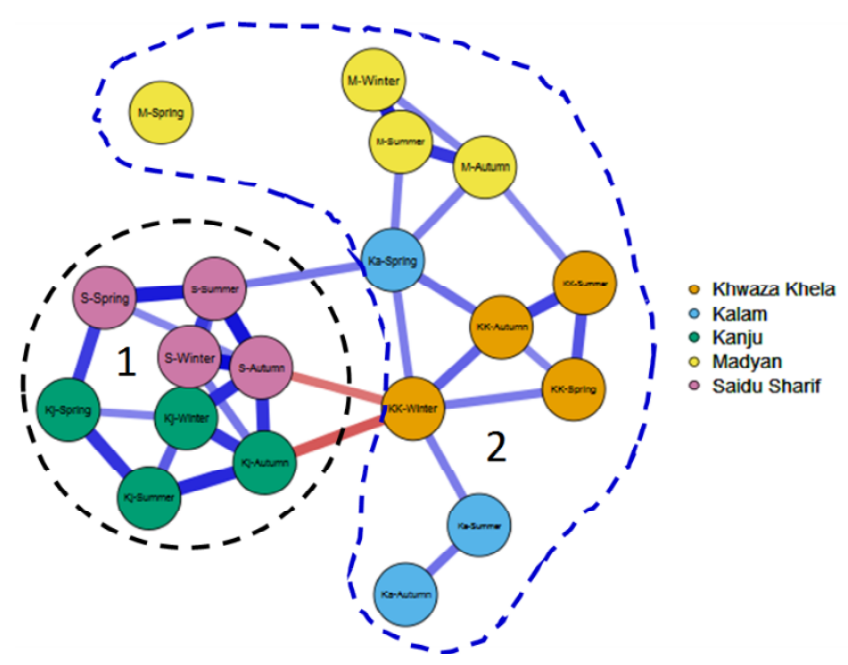

Fig. 4. Correlation plot of species richness of algal communities in the Swat River valley on the level of $40 \%$ similarity. Legend: studied sites, 1 - first correlation core, 2 - second correlation core

Рис. 4. Корреляционный граф видового богатства водорослевых сообществ долины р. Сват на уровне $40 \%$ сходства. Условные обозначения: исследованные участки, 1 - первое корреляционное ядро, 2 - второе корреляционное ядро

Correlation of seasonal communities in fig. 4 shows no relation with the season. However, communities in the first core represent full diversity from two lower sites (Saidu Sharif and Kanju) only. All the other seasonal communities are combined in core 2 . Distribution in fig. 4 and 5 demonstrates that altitude of the monitoring site is a regulating factor of algal diversity development.

In order to develop a statistical approach for algal and environmental variables relationships, 3D surface plots of species richness in algal communities' relationships and major environmental variables were 


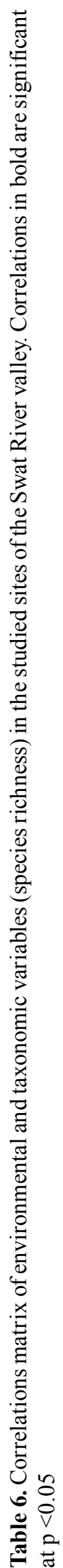

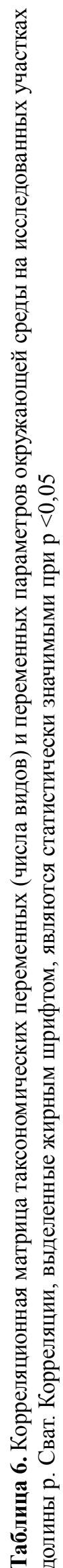

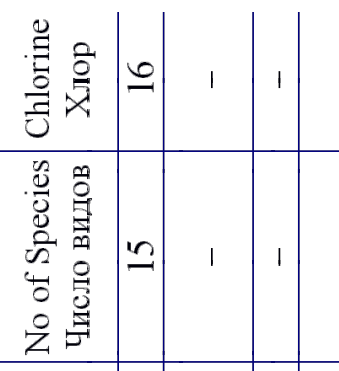

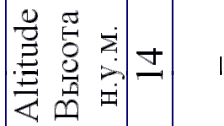

\begin{tabular}{|c|c|c|c|c|c|c|c|c|c|c|c|c|c|c|}
\hline 교 & 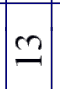 & 1 & 1 & 1 & 1 & 1 & I & 1 & 1 & 1 & 1 & 1 & - & $\begin{array}{l}\infty \\
n \\
? \\
?\end{array}$ \\
\hline I0工工 & $\simeq$ & 1 & 1 & 1 & 1 & I & I & 1 & 1 & 1 & 1 & - & 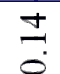 & ర్ర \\
\hline$\vec{U}$ & $\exists$ & 1 & 1 & I & 1 & I & 1 & 1 & 1 & 1 & - & $\tilde{n}$ & $\infty$ & กै \\
\hline
\end{tabular}

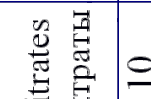

苦急

$-$\begin{tabular}{ll|l|l|l}
0 & $\infty$ & $\infty$ & 0 & $\infty$ \\
0 & $\stackrel{0}{0}$ & $\stackrel{0}{0}$ & $\stackrel{1}{i}$
\end{tabular}

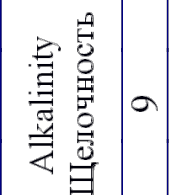

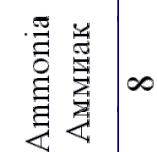

○苔

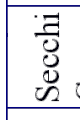

\begin{tabular}{|c|c|c|c|c|c|c|c|c|c|c|c|c|c|c|c|c|c|c|c|}
\hline 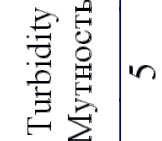 & $n$ & 1 & 1 & & I & - & $\frac{0}{\stackrel{0}{i}}$ & & $\begin{array}{l}0 \\
0 \\
0\end{array}$ & $\stackrel{g}{b}$ & & & & & & & & & \\
\hline
\end{tabular}

E

管

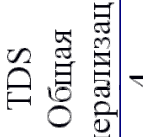

(1)

$\checkmark$

\begin{tabular}{|c|c|c|c|c|}
\hline I & $\mathrm{m}$ & & - & $\stackrel{2}{\circ}$ \\
\hline 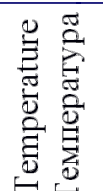 & N & - & $\stackrel{T}{0}$ & $\begin{array}{l}10 \\
0 \\
0\end{array}$ \\
\hline
\end{tabular}

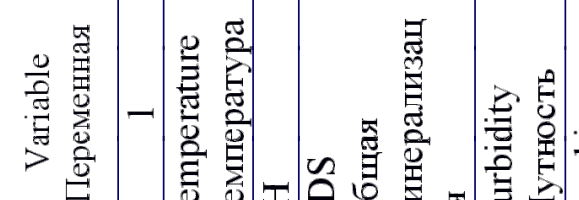

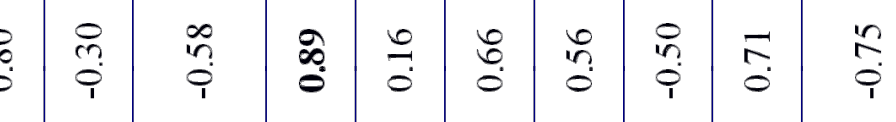

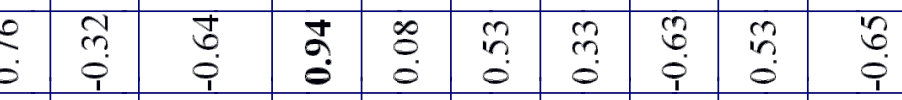

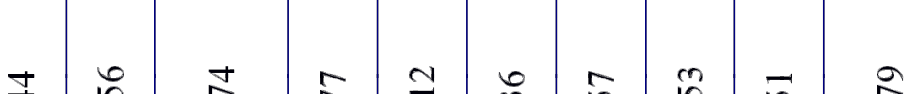

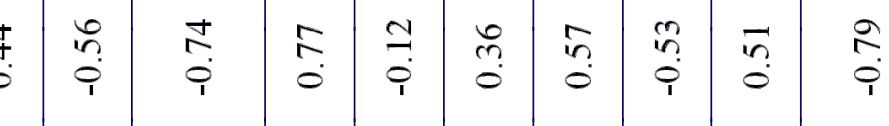




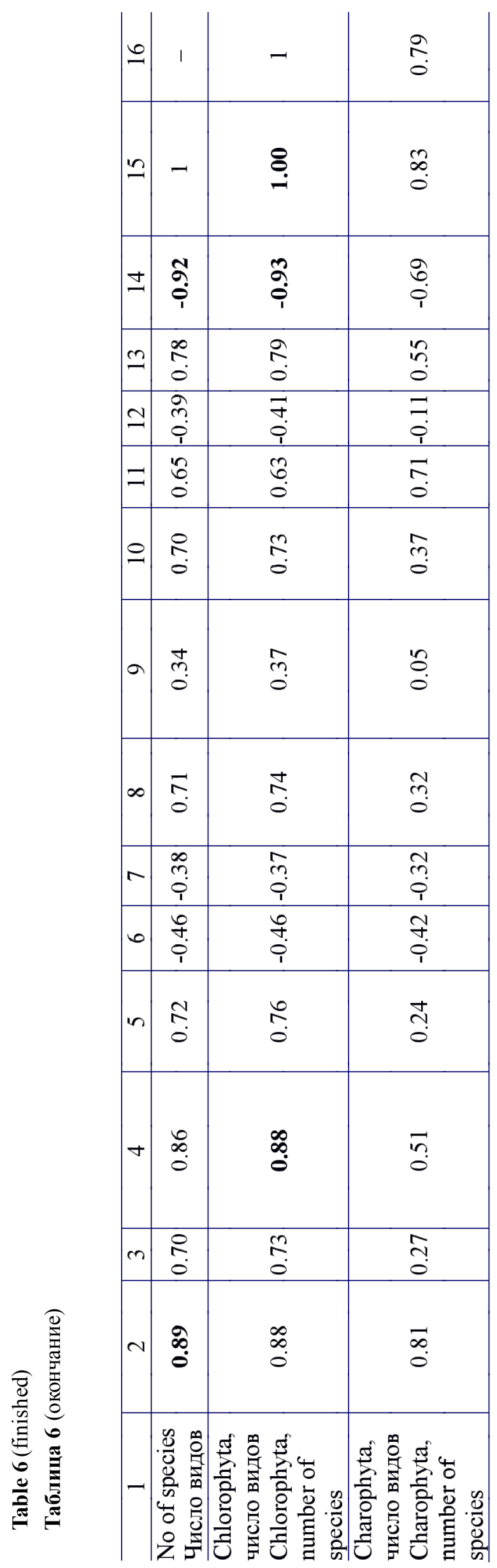




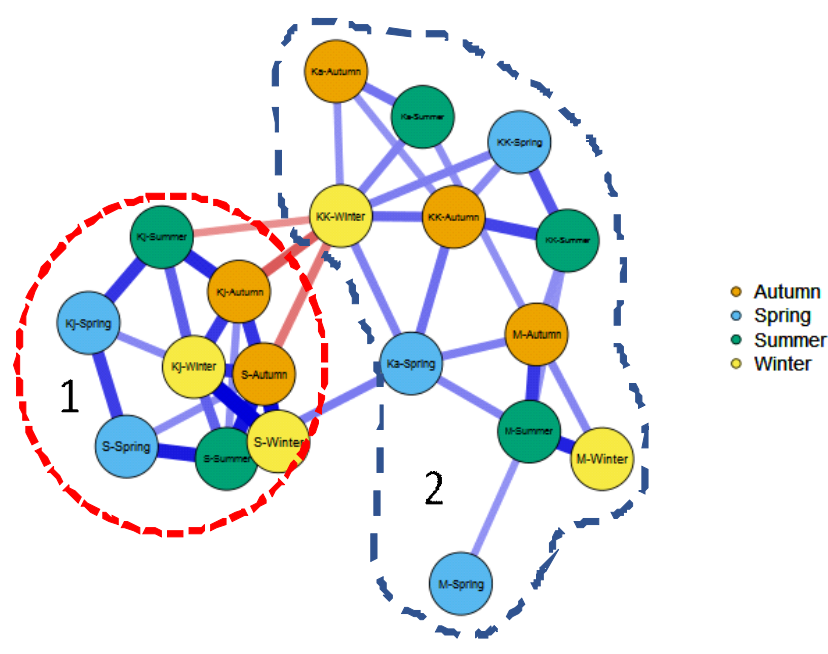

Fig. 5. Correlation plot of seasonal variations of species richness of algal communities in the Swat River valley on the level of $40 \%$ similarity. Legend: seasons; 1 - first correlation core; 2 - second correlation core

Рис. 5. Корреляционный граф видового богатства водорослевых сообществ долины р. Сват на уровне 40 \% сходства. Условные обозначения: сезоны; 1 - первое корреляционное ядро, 2 - второе корреляционное ядро constructed. Fig. 6 shows that the site altitude is a more pronounced regulating factor of species richness than dissolved oxygen (a), but water temperature stimulated species richness, whereas altitude suppressed it (b). Calculations for all other factors showed no significant dependencies.

\section{CONCLUSION}

The results of this research show that altogether 105 taxa of algae from Charophyta and Chlorophyta taxonomic divisions were identified in 200 samples of algae collected from five sites in the Swat River Basin during 2007-2016. Chlorophyta species number has strongly prevailed with 86 taxa as compared to Charophyta (19 taxa). For the first time, this diversity has been revealed for the streams of high mountain habitats of the Swat River Basin that is the upper part of the Indus River. The altitude of the studied sites varied from 972 to $2,061 \mathrm{~m}$ a.s.1. Water temperature, $\mathrm{pH}$, TDS, ammonia, nitrate concentration, and zinc decreased with increasing of altitude, whereas water turbidity, water transparency, and iron increased. The

\section{D Surface Plot of No of Species against Altitude and DO Spreadsheet1 $17 \mathrm{v}^{*} 10 \mathrm{c}$}

No of Species $=105.2916-0.0223^{*} x-1.3532^{*} y$
3D Surface Plot of No of Species against Altitude and Temperature Spreadsheet $17 \mathrm{v}^{*} 10 \mathrm{c}$

No of Species $=66.4603-0.0145^{*} \mathrm{x}+2.3658^{*} \mathrm{y}$
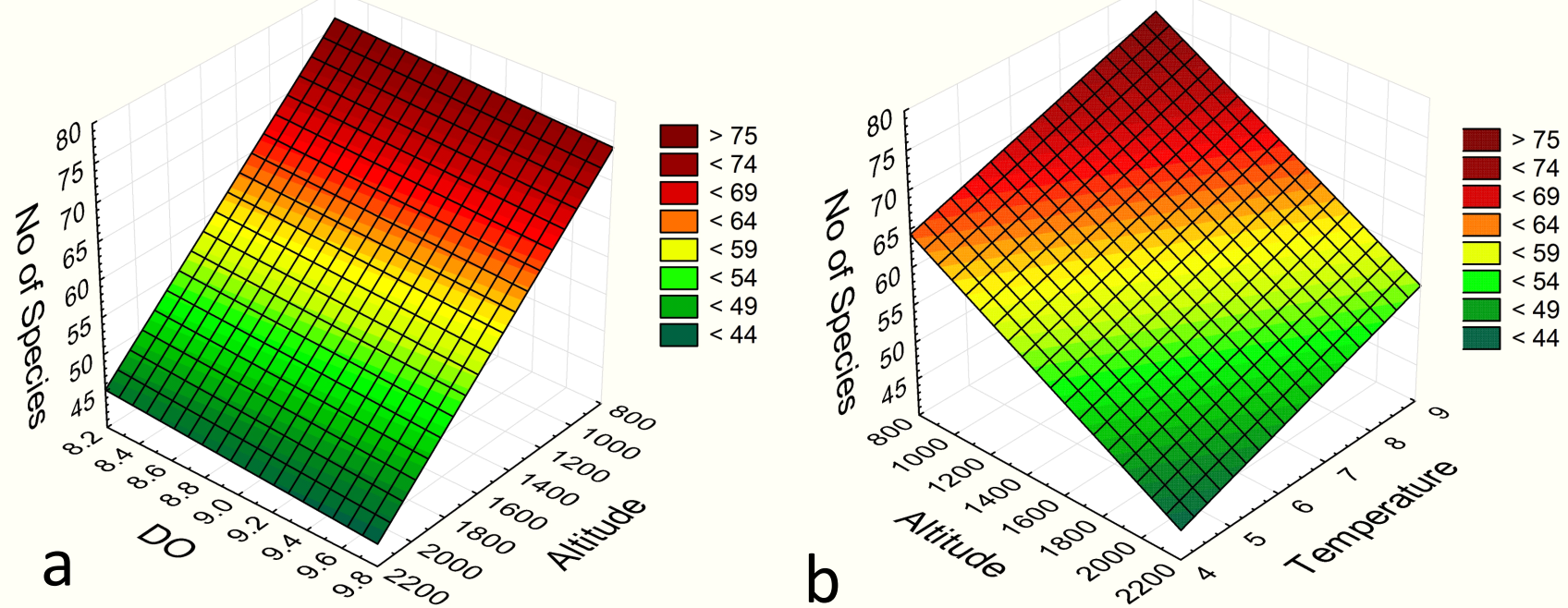

Fig. 6. 3D surface plots of the relation of species richness of algal communities in the Swat River valley to site altitude and dissolved oxygen (a), and to water temperature (b)

Рис. 6. Объемные поверхностные диаграммы зависимости видового богатства водорослевых сообществ долины р. Сват от высоты участка над уровнем моря и содержания растворенного кислорода (а) и от температуры воды (b) 
head part of the studied algal diversity, which contains over $50 \%$ of species, is represented by 19 genera, out of which only two genera belonged to Charophyta (11 species, or $10.4 \%$ ) but all other ones were affiliated to Chlorophyta algae. Distribution of the types of thallus structure allowed for the assumption that most of the revealed algal communities have a lowly organized structure and, therefore, developed in an unstable environment. The decrease of total species richness as well as Chlorophyta and Charophyta species number with an increase of the site altitude were revealed in the seasonal dynamics of the studied species richness. It allowed to conclude that altitude can be a strong regulating factor for algal community growth in the Swat River Basin. Statistical analysis of relationships of algal species richness and environmental variables also suggests that altitude of the monitoring site is a regulating factor of algal diversity development, as well as that water temperature stimulated species richness, whereas altitude suppressed it. Low water temperatures are unfavorable for the growth of algae in general and Chlorophyta algae in particular. Therefore, mild summer and pleasant spring seasons result in prolific vegetative growth of Chlorophyta and Charophyta species of algae in the Swat River Basin habitats, but algal species here can be affected by the pollution impact because they are represented by lowly organized thallus structure. The study can help in framing strategies for conservation of the aquatic ecosystems of the upper Swat River Basin in Pakistan.

\section{ACKNOWLEDGEMENTS}

The work was partly supported by the Israeli Ministry of Aliyah and Integration.

\section{REFERENCES}

1. Barinova S., Ali N., Barkatullah, Sarim F.M. Ecological adaptation to altitude of algal communities in the Swat Valley (Hindu Cush Mountains, Pakistan). Expert Opinion on Environmental Biology, 2013, no. 2 (2), pp. 1-15. doi: 10.4172/2325-9655.1000104.

2. Khuram I., Ahmad N., Jan S., Barinova S. Freshwater green algal biofouling of boats in the Kabul River, Pakistan. Oceanological and Hydrobiological Studies, 2014, no. 43 (4), pp. 329-336. doi: 10.2478/s13545-0140150-y.

3. Barinova S., Khuram I., Asadullah, Ahmad N., Jan S., Shin D.H. How water quality in the Kabul River, Pakistan, can be determined with algal bio-indication. Advance Studies in Biology, 2016, vol. 8, no. 8 (4), pp. 151-171.
4. Khuram I., Barinova S., Ahmad N., Ullah A., Ud Din S., Jan S., Hamayun M. Ecological assessment of water quality in the Kabul River, Pakistan, using statistical methods. Oceanological and Hydrobiological Studies, 2017, no. 46(2), pp. 140-153. doi:10.1515/ohs-2017-0015.

5. Khuram I., Muhammad Z., Ahmad N., Ullah R., Barinova S. Green and Charophyte algae in bioindication of water quality of the Shah Alam River (District Peshawar, Pakistan). Transylvanian Review of Systematical and Ecological Research, "The Wetlands Diversity”, 2019, no. 21.1, pp. 1-16. doi: 10.2478/trser2019-0001.

6. Mursaleen, Shah S.Z., Ali L., Ahmad N., Kuram I., Barinova S.S. Algal communities of the Mardan River in ecological assessment of water quality in district Mardan, Pakistan. MOJ Ecology \& Environmental Sciences, 2018, vol. 3, no. 2, pp. 82-92. doi: 10.15406/ mojes.2018.03.00071.

7. Mursaleen, Shah S.Z., Ali L., Ahmad N., Khuram I., Barinova S. Bioindication of water quality by algal communities in the Mardan River, Pakistan. International Journal of Biology and Chemistry, 2018, no. 11 (1), pp. 65-81.

8. Ali L., Shah S.Z., Mursaleen, Khuram I., Ahmad N., Barinova S.S. Response of algal diversity to physicochemical parameters of polluted waters in spring low water season in District Charsadda, Pakistan. Aquatic Bioresources \& Environment, 2018, vol. 1, no. 3-4, pp. $45-55$.

9. Wehr J.D., Sheath R., Kociolek J.P. Freshwater algae of North America: ecology and classification. Cambridge: Academic Press, 2002, 1066 p.

10. Algal ecology: freshwater benthic ecosystem. R.J. Stevenson, M.L. Bothwell, R.L. Lowe, J.H. Thorp. (Eds.). Cambridge: Academic Press, 1973, 753 p.

11. Barinova S.S., Medvedeva L.A., Anisimova O.V. Bioraznoobrazie vodorosley-indikatorov okruzhayushchey sredy [Diversity of algal indicators in the environmental assessment]. Tel Aviv: Pilies Studio Publisher, 2006, 498 p. (In Russian).

12. Barinova S.S., Bilous O.P., Tsarenko P.M. Al'goindikatsiya vodnykh ob"yektov Ukrainy: metody $\mathrm{i}$ perspektivy [Algal indication of water bodies in Ukraine: methods and perspectives]. Haifa-Kiev: University of Haifa Publ., 2019, 367 p. (In Russian).

13. Shrestha G., Rai S.K. Algal flora of Rajarani Lake, Dhankuta and their seasonal distribution. Our Nature, 2017, no. 15 (1-2), pp. 44-54. doi: 10.3126/on.v15i12.18793 .

14. Soni H., Thomas S. Associative dependence among plankton and macrophytes as pollution markers at tropical lentic environ, Gujarat, India. International Journal of Environment, 2014, no. 3 (2), pp. 175-191. doi: 10.3126/ ije.v3i2.10528.

15. Shams M., Afsharzadeh S., Atici T. Seasonal variation in phytoplankton communities in Zayandeh-Rood Dam 
Lake (Isfahan, Iran). Turkish Journal of Botany, 2012, no. 36, pp. 715-726. doi: 10.3906/bot-1104-19.

16. Asghar A., Shinwari Z.K., Sarim F.M. Contribution to the algal flora (Chlorophyta) of fresh waters of District Swat. N.W.F.P., Pakistan. Pakistan Journal of Botany, 2010, no. 42 (5), pp. 3457-3462.

17. Sarim F.M., Sultana N., Nisa K. Freshwater algae of River Sardaryab, District Charsadda, Pakistan. Pakistan Journal of Plant Sciences, 2009, no. 15, pp. 69-74.

18. Reshmi S. Chlorophycean biodiversity in Wetlands of Satna (M.P.), India. Biodiversity and Environment, 2004, pp. 171-190.

19. Leghari M.K., Waheed S.B., Leghorn M.K. Ecological study of algal flora of Kunhar River of Pakistan. Pakistan Journal of Botany, 2001, no. 33, pp. 629-636.

20. Leghari M.K, Shah M., Leghari M.Y. Ecological study of blue-green algae from Rawal Dam Water, Islamabad. International Journal of Phycology and Phycochemistry, 2005, no. 1, pp. 5-12.

21. Ertan O.O., Morkoyunlu A. The algae flora of Aksu Stream (Isparta - Turkey). Turkish Journal of Botany, 1998, no. 22, pp. 239-256.

22. Department of Geography and Urban Planning. District census report. Peshawar: University of Peshawar Press, 1998.

23. Standard methods for the examination of water and wastewater. 22 ${ }^{\text {rd }}$ ed. E.W. Rice, R.B. Baird, A.D. Eaton, L.S. Clesceri. (Eds.). Washington: Water Environment Federation (APHA) American Public Health Association Publ., 2012, 1496 p.

24. Edler L., Elbrächter M. The Utermöhl method for quantitative phytoplankton analysis. In: Microscopic and molecular methods for quantitative phytoplankton analysis. B. Karlston, C. Cusack, E. Bresnan. (Eds.). Paris: UNESCO Publ., 2010, pp. 13-21.

25. Akiyama M., Hirose H., Yamagishi T., Hirano M. Class Chlorophyceae. In: Nihon tansuisõzukan [Illustrations of the Japanese fresh-water algae]. H. Hirose,
T. Yamagishi, M. Akiyama. (Eds.). Tokyo: Uchida Rokakuho Publishing, 1977, pp. 275-760. (In Japanese).

26. Tiffany L.H., Britton M.E. The algae of Illinois. Chicago: University of Chicago Press, 1952, 407 p.

27. Siddiqi I.I., Faridi M.A.F. The Chlorococcales of Peshawar valley. Biologia, 1964, no. 10, pp. 1-88.

28. Prescott G.W. Algae of the Western Great Lake area. East Lansing: Michigan State University Publ., 1961, $975 \mathrm{p}$.

29. Smith G.M. The fresh-water algae of the United States. New York: McGraw-Hill Book Company, 1950, 719 p.

30. Guiry M.D., Guiry G.M. AlgaeBase. World-wide electronic publication. Galway: National University of Ireland Press, 2018. Available at: http:// www.algaebase.org (accessed 24.01.2019).

31. Love J., Selker R., Marsman M., Jamil T., Dropmann D., Verhagen A.J., Ly A., Gronau Q.F., Smira M., Epskamp S., Matzke D., Wild A., Rouder J.N., Morey R.D., Wagenmakers E.J. JASP: graphical statistical software for common statistical designs. Journal of Statistical Software, 2019, vol. 88, no. 2, pp. 1-17. doi:10.18637/jss.v088.i02.

32. Barinova S. Systemic criteria for the analysis of alphaand gamma-diversity of freshwater algae. International Journal of Environmental Sciences \& Natural Resources, 2017, vol. 4, issue 2, pp. 555-633 (1-6). doi: 10.19080/ IJESNR.2017.04.555633.

33. Haroon K., Fiaz M., Khan S., Hussain F., Shah S.Z., Shah M., Shoaib M., Raza F., Leghari M.K. Taxonomic study of freshwater green algae in relation to water quality of Tehsil Landikotal, Khyber Agency, Pakistan. Pure and Applied Biology, 2017, vol. 6, no. 4, pp. 1328-1334.

34. Solak C.N., Barinova S., Ács E., Dayioğlu H. Diversity and ecology of diatoms from Felent creek (Sakarya river basin), Turkey. Turkish Journal of Botany, 2012, no. 36, pp. 191-203.

Поступила 03.05.2019

Принята к печати 28.06.2019 